\title{
Design Management System of OTEC Plant Based on a Knowledge Template*
}

\author{
Tsuyoshi KOGA $^{* *}$, Norihiko GOTO**, Shin OKAMURA ${ }^{* * *}$ \\ and Kazuhiro AOYAMA** \\ **Department of Systems Innovation, School of Engineering, the University of Tokyo, \\ 7-3-1, Hongo Bunkyo-ku, Tokyo 113-8656, Japan \\ E-mail: koga@sys.t.u-tokyo.ac.jp \\ ${ }^{* * *}$ Xenesys Inc. \\ 12-16-7F, Mita Minato-ku, Tokyo 108-8328, Japan
}

\begin{abstract}
We focused on developing a management system that provides a design process in which the important attributes of the product can be easily incorporated. We developed a knowledge template to express the product information using five types of knowledge, namely, knowledge about product structure, product entity, product function, product constraint, and product design process. To determine a suitable design process, we first compared the knowledge template of existing products with the product being designed. We calculated the consistency between the two models. From the results of the consistency calculation, we selected and extracted the available knowledge. We created a new design process by using the knowledge extracted from the design template. Finally, we evaluated the process from three perspectives: the ease with which the customer requirements could be reflected, the level of difficulty of the design conflict problem, and the level of difficulty of the design loop problem. Based on the results of this evaluation, designers can select a process to design a new product. We developed a prototype system and used it to design an ocean thermal energy conversion (OTEC) system. The design knowledge for an actual OTEC design was adequately represented by the proposed knowledge template. The most important attribute has a higher value in the design process obtained using the proposed management system process than in the existing system. The proposed management system yields a design process that can easily reflect customer requirements.
\end{abstract}

Key words: Plant Modeling, Template Design, Flexible Knowledge Template, Design Process Navigation, Ocean Thermal Energy Conversion

\section{Introduction}

\subsection{Product design based on knowledge template}

Current plant system design is focused on model change, wherein a new model is produced by improving the existing products, and the idea of using past design knowledge to make modifications to existing products is fast gaining ground. Template design is one such idea that makes full use of past design knowledge. This paper defines past design knowledge as a "knowledge template." By using the knowledge template, a designer can design a new plant system simply by improving the existing system. Also, designers can design a common knowledge template from which a new product can be developed

${ }^{*}$ Received 2 June, 2009 (No. 09-0229) [DOI: 10.1299/jamdsm.3.257] 
according to a customer's requirements. This design method is called template-based design. The use of a common knowledge template has the following advantages.

(1) Design efficiency is promoted

In template-based design, designers start working from a common template. Since the base of the product is already complete, the designers can focus on other tasks.

(2) Sharing common parts across several products leads to cost reduction.

(3) Sharing design knowledge becomes easier.

Most of the existing design knowledge is in the form of human experience. This type of knowledge can easily be lost; further, knowledge sharing becomes difficult if there are more than two designers involved. By arranging the design knowledge in the form of a template, these problems can be solved.

In recent times, there have been several studies on template-based design support. Hannah defined design templates as reusable design process modules that support decision-making at various stages in the design process ${ }^{(1)}$. Abbott focused on the importance of functional templates and showed that a template tool for functional modeling helps a user develop modeling skills even if he/she does not possess extensive background knowledge ${ }^{(2)(3)}$. Nonetheless, there are three difficulties involved in template-based design.

First, it is difficult to produce the common knowledge template. Since many products have to be produced from one template, the design template should be versatile, flexible, and adaptable. If the template lacks sufficient adaptability, the expected design efficiency will not be achieved. Second, it is difficult to express the unclear knowledge that designers possess. As mentioned previously, most design knowledge is in the form of the designer's experience. For design knowledge to be shared, it is essential that it be described clearly. However, most design knowledge is fuzzy and designers cannot describe this knowledge with enough clarity for it to be shared.

Third, it is difficult to derive a flexible design process. A "flexible" design process is one that can easily be selected according to the needs of the customer(s) or the technological requirements of the product. The procedure that is currently followed in product design in order to select the design process and the order in which designers decide the product attributes is complicated and requires considerable time and expense. In addition, this process is so unclear that even the designers themselves do not know how they have derived a particular design process. This is particularly true when designing a large-scale product such as plants and ships. Therefore, the final design process selected is not always the "easiest" process; in other words, the final process may not be the one that allows designers to complete the design under fewer constraints.

\subsection{Approaches and research purpose}

As mentioned in the previous section, it requires considerable energy and time to determine which attribute of the product to begin with. We decided to make this process efficient and therefore set two goals to achieve the same. The first is to propose a knowledge template model from which designers can extract information in order to derive the design process. The second is to use the template to guide a design process that can easily reflect the requirements or constraints of a product. According to K. Otomi ${ }^{(4)}$, the opportunity for cost reduction is the greatest in conceptual design. Therefore, it is reasonable to apply template design to the early stage of design.

The overview of the research purpose is shown in Fig. 1. In the current design process, planning is very difficult because the planning process is complex and the different processes are interlinked with each other. A designer must consider various design requirements; hence, it is difficult to unravel and the designer should be highly skilled. Herein, we propose a model of a knowledge template for representing past design information; this knowledge template is meant to help a designer to select a suitable design 


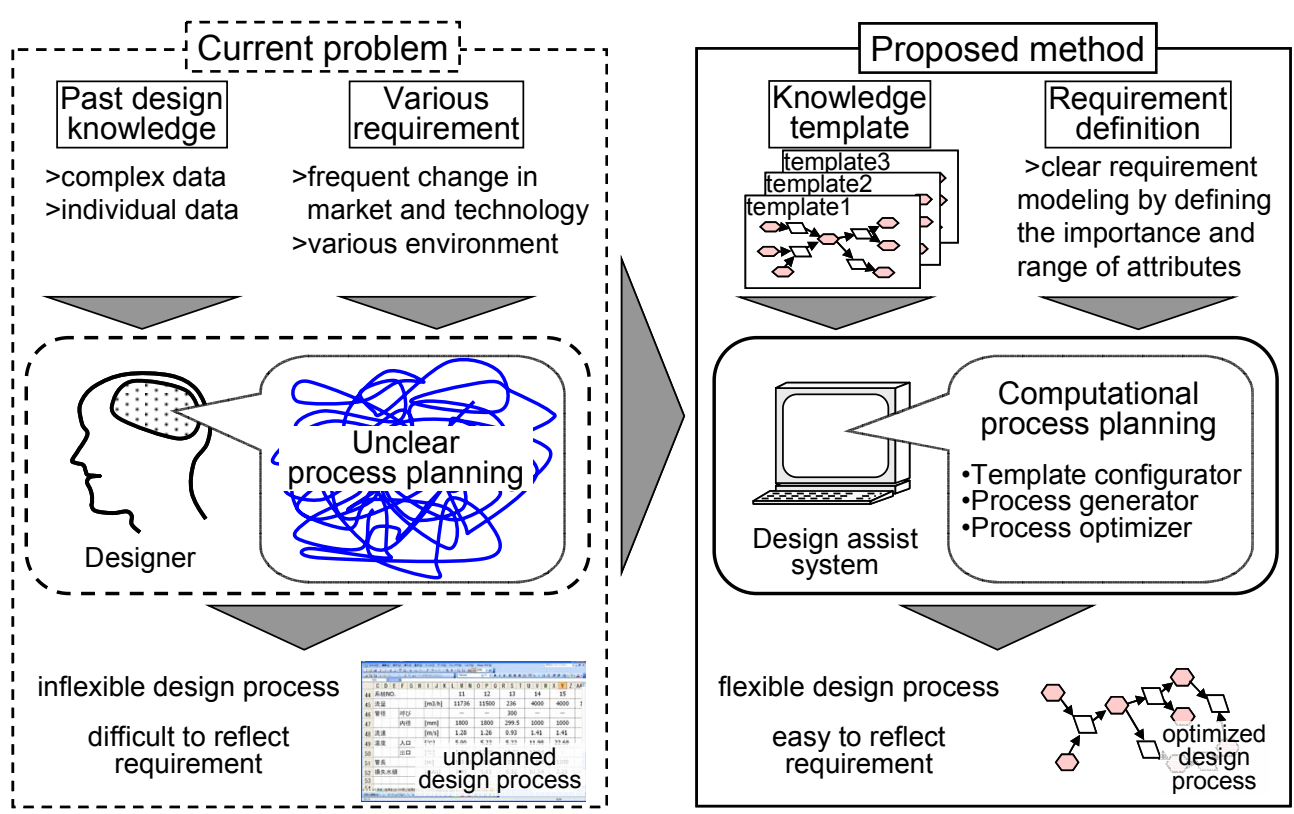

Fig. 1 Overview of research purpose: current problem and proposed method

process in accordance with the various requirements and constraints. Our objective is to develop a design guide system on the basis of the process determined from the knowledge template and the product requirements by following detailed goals (A) and (B):

(A) proposition of a guide method to provide a design process in which the important attributes of the product can be easily incorporated by using the knowledge extracted from the design template, and

(B) effectiveness validation of proposed method by developing a prototype system and designing an ocean thermal energy conversion (OTEC) system.

\section{Model of Design Knowledge Template and the Design Process}

\subsection{Design knowledge}

To achieve our first goal — namely, to propose a knowledge template model from which designers can extract information in order to derive the design process - the complete design knowledge must be suitably arranged. In this section, we explain the knowledge that designers deal with and how this knowledge is expressed in the template model.

In this research, design knowledge has been classified into five types: knowledge about product structure, knowledge about product hierarchy, knowledge about product function, knowledge about product constraints, and knowledge about design process.

\subsection{Model of the design knowledge template}

We have developed a design support system that makes use of six elements-entity, interface, attribute, constraint, hierarchy link, and calculation flow-to express the five types of knowledge. To explain how design knowledge is described, we have provided an example of an intake system (see Fig. 2). Let us understand each of the six elements by considering the example of the intake system.

The elements "entity" and "interface" are used to describe the knowledge about the product structure. An intake system comprises a pump and a pipe; therefore, design knowledge about the product structure can be described by the entities "pump" and "pipe." The relationship between the pump and pipe is described by an interface. The element "hierarchy link" is used to describe the knowledge about the product hierarchy. In the case of the intake system, "hierarchy link" describes the refined structure of the pump and pipe. 


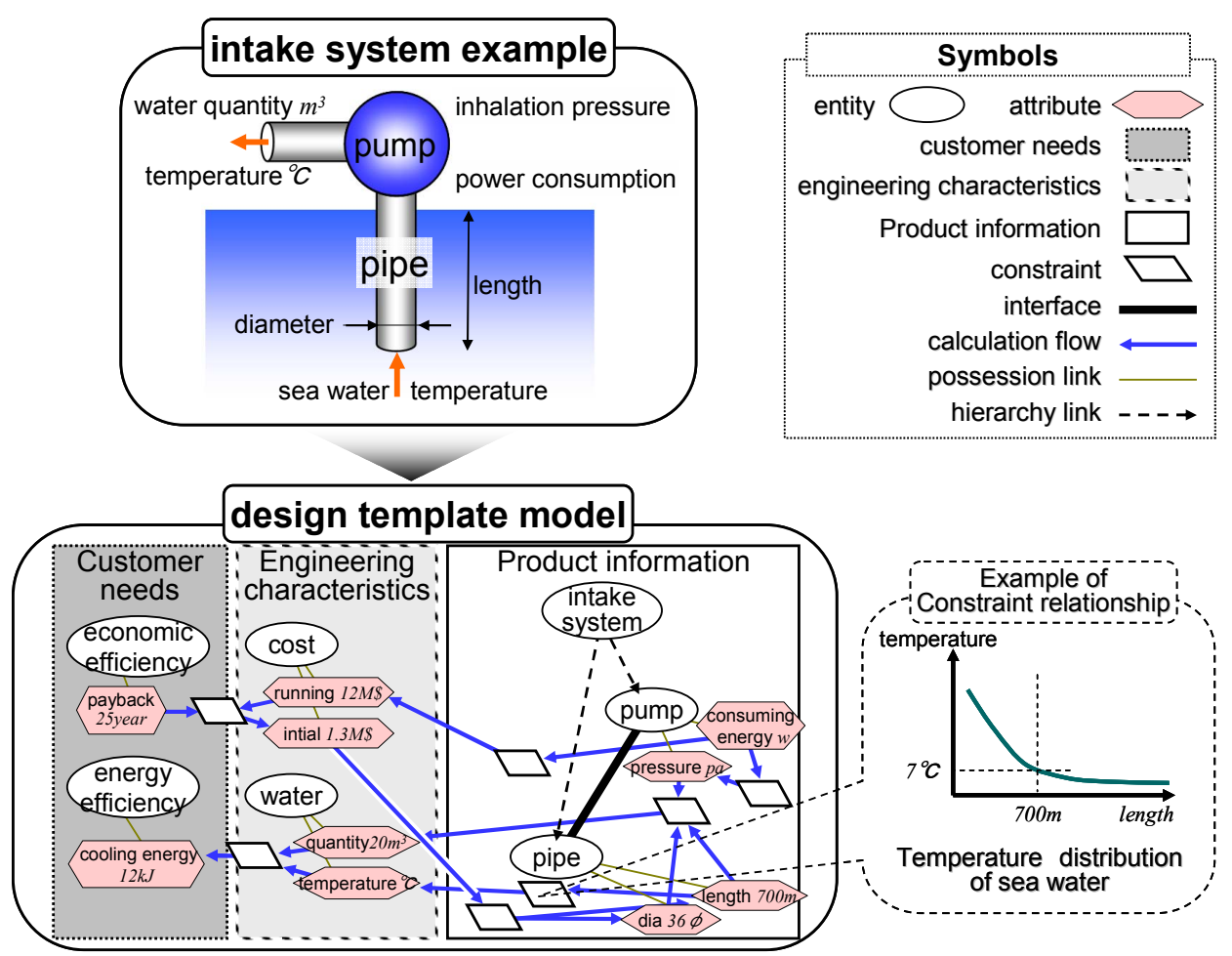

Fig. 2 Model of the design knowledge template -an example of a deep water intake system-

The elements "customer needs layer" and "engineering characteristics layer" are used to describe the knowledge about the product function. With regard to the intake system, "energy" and "economic efficiency" have been described as customer needs. The corresponding engineering characteristics required to meet the customer needs are "cost" and "water," as defined in the engineering characteristics layer. The elements "attribute" and "constraint" are used to describe the knowledge about product constraints. In the intake system example, there exists a constraint with regard to "water temperature" and "pipe length": the longer the length, the lower is the water temperature. The element "calculation flow" is used to describe the knowledge about the design process. In the intake system example, the attribute "water temperature" can be determined from the "pipe length" and there exists a constraint between these two attributes. The combination of these calculation flows enables us to express the design process (Fig. 3). On the basis of the proposed knowledge template model, we developed a design support system.

In order to confirm whether the developed knowledge template is capable of representing an actual complex product, we applied this template to the design of an ocean thermal energy conversion (OTEC) plant. The spreadsheet that is actually used to design an OTEC plant was chosen. As a result, the proposed knowledge template could express all the


Fig. 3 Design process representation based on knowledge template 

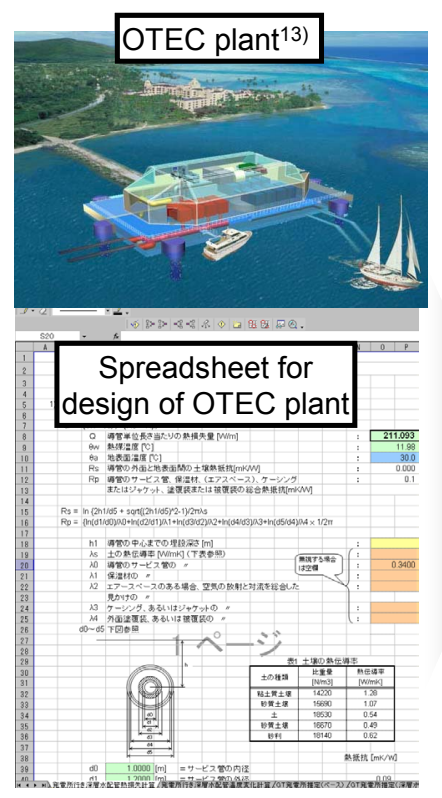

OTEC plant representation using design system (knowledge template)

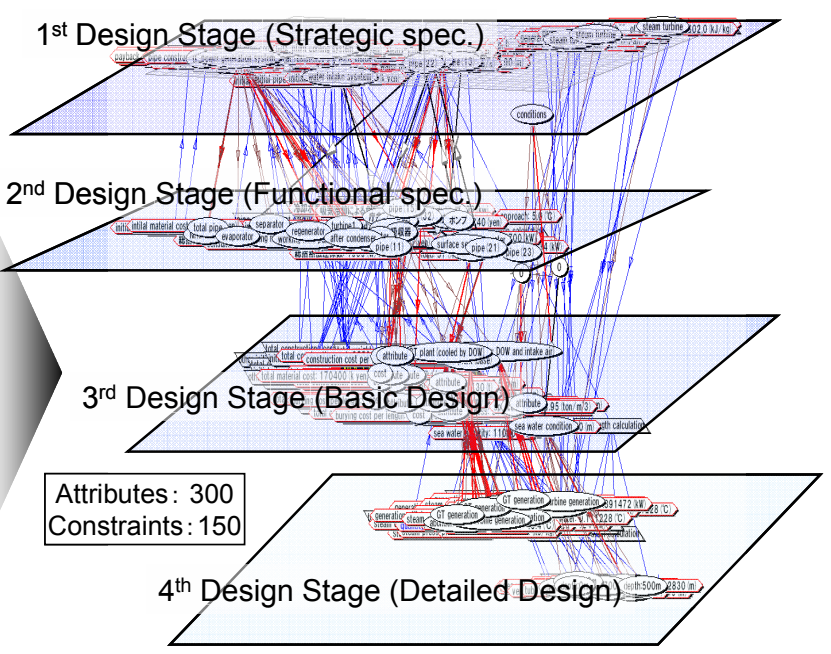

Fig. 4 OTEC plant representation using knowledge template

elements described in the spreadsheet. From this result, it can be said that the necessary design knowledge can be expressed by this system. The result of the design example is shown in Fig. 4. However, this does not imply that this system can express all the knowledge that designers possess. There still remains a significant amount of implicit knowledge.

\section{Generation of Design Process from Knowledge Template}

The second goal of this research is to use the knowledge template to guide a design process that can easily reflect the requirements or constraints of a product. The following steps are proposed in order to achieve this goal.

1. Select the template according to consistency (that is, the new product description should be consistent with the template data).

2. Extract information about the design process from the template that is "available."

3. Combine the extracted knowledge to create a new process.

4. Evaluate the process difficulty from three perspectives: importance, design loop, and design conflict.

\subsection{Description of product information}

Initially, the designer must describe the information about the new product by using the proposed design system. To describe the product information, the designer can use the existing template data, thereby saving both time and cost. In case the designer uses the template data, the designer must first choose the template to refer to. Next, the designer must arrange the information according to the new product that he or she wants to design. After describing the product information, the designer must set the customer's requirements by changing the importance of the relevant attributes. At this stage, the calculation flows have no direction and thus the design process is undecided.

\subsection{Selecting knowledge template}

Next, we compare the new product description with the template data and calculate the consistency of the template. The consistency is calculated on the basis of two perspectives. They are described below. 
(1) Consistency in structure: The higher the proportion of entities in the new product description that correspond with the entities in the template, the higher is the consistency of that template.

(2) Consistency in terms of level of importance: The closer the importance of an attribute in the new product description is to the importance of the same attribute in the template, the higher is the consistency of that template.

Higher consistency implies that the template is similar to the structure of the product to be designed or that it matches the requirements the product attempts to satisfy.

\subsection{Extracting product information and creating design process from template}

Once the designer has selected a template on the basis of its consistency, the available information is extracted in order to produce the candidate processes.

First, we compare each constraint in the description of the new product with each constraint in the template and choose the "same" constraints from the template. Here, a "same" constraint is one that has the same set of input and output attributes. Further, the attribute's identity is judged on the basis of the attribute itself and the entity the attribute belongs to. Further, when the attribute identity is judged, the attribute value is not considered because it has very less importance in the determination of the design process. In this research, it is assumed that the values of the attributes are decided after the design process is fixed. The process of extracting the available constraints is shown in Fig. 5.

If some of the constraints in the description of the new product are not filled with the information extracted from the selected templates, then the designers have to fill in the constraints. In the next stage, we produce the candidate processes by combining the "same" constraints that have been selected. In case the designer has described the information in the constraints, that information is also used to produce candidate processes.

\subsection{Evaluating difficulty of design process}

This research proposes new metrics to evaluate the difficulty of the design process. These metrics balance the importance of the attribute and the range of the attribute, considering design loop and design conflict. In addition, this paper adds the importance and range of the attribute based on our previous propositions ${ }^{(5)}$.

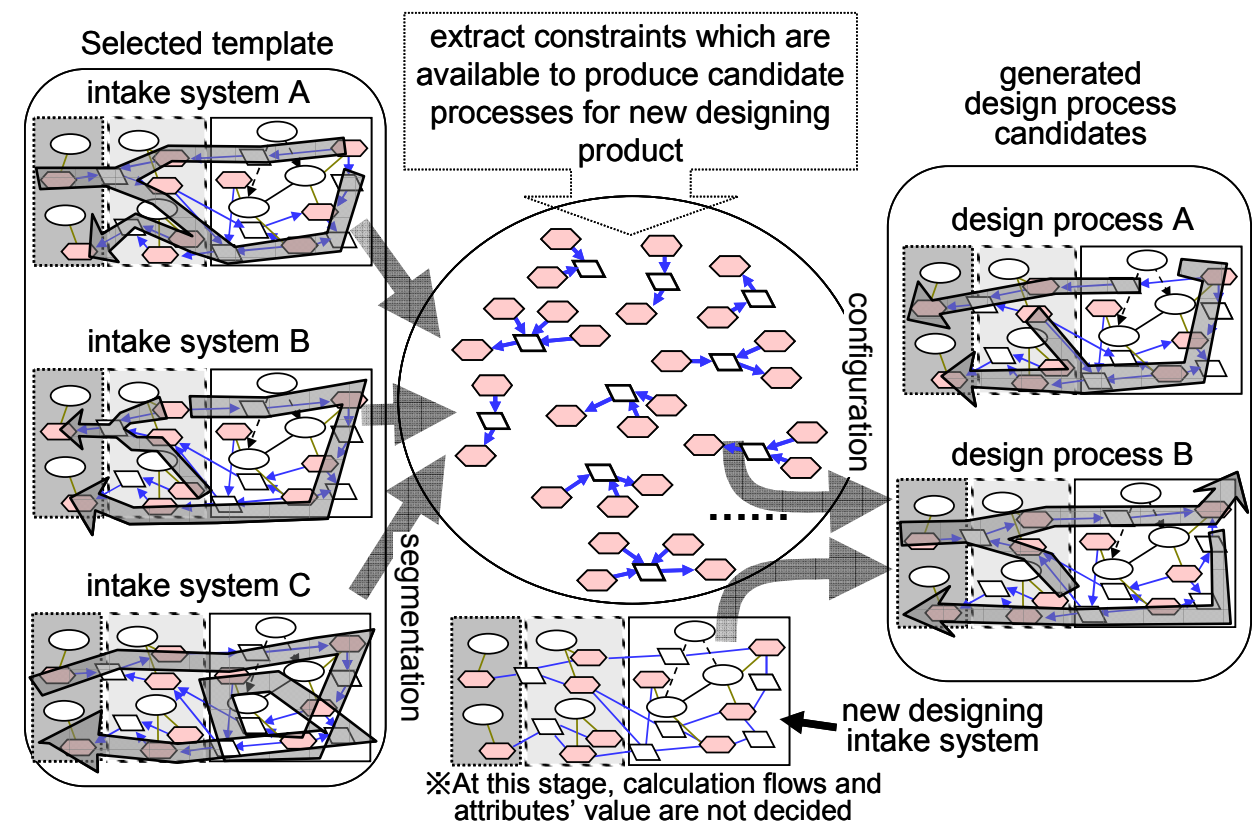

Fig. 5 Extraction of product information and creation of design process from knowledge template (model element is defined as in Fig.2) 
The case in which the designers have to determine the attribute values by checking the related attributes is known as the design loop problem. The difficulty involved in solving the design loop problem is expected to increase with the number of related attributes. In this research, the difficulty of the design loop as the square of the number of related attributes. This estimation is same as the area of loop in DSM, which is consistent with the other existing researches ${ }^{(6)-(10)}$.

Conflict is a problem wherein designers have to determine one attribute under more than one constraint. The difficulty involved in solving the conflict problem is expected to increase with the numbers of related constraints. The conflict size is the number of related attributes. However, the equations used to evaluate the loop difficulty and conflict difficulty are different from the equations used to determine the actual design difficulty in that they do not consider the acceptable values for the related attributes. However, other studies on the design process, such as those of Yoshikawa ${ }^{(11)}$ and Clarkson ${ }^{(9)}$, also do not consider acceptable values for the attributes.

In this research, the range of acceptable values of attribute $i$ is defined as follows.

$$
r_{i}=\frac{r_{i}^{\max }-r_{i}^{\min }}{A_{i}}
$$

where:

$r_{i}$ : range of attribute $i, r_{i}^{\max }:$ maximum value of attribute $i, r_{i}^{\min }:$ minimum value of attribute $i, \mathrm{~A}_{i}$ : average of $r_{i}^{\max }$ and $r_{i}^{\min }$

In this research, the loop difficulty and conflict difficulty ${ }^{(5)}$ are redefined considering the range of an attribute.

$$
\begin{aligned}
& D_{l}=l_{x}^{2} / \prod_{i=1}^{l_{x}} r_{i} \\
& D_{c}=c_{s}^{3} / r_{i}
\end{aligned}
$$

where:

$D_{l}$ : loop difficulty, $l_{s}$ : loop size, $r_{i}$ : range of attribute $i$ that comprises the loop,

$D_{c}$ : conflict difficulty, $\mathrm{c}_{s}$ : conflict size, $r_{i}$ : range of attribute $i$ that comprises the conflict

Loop and conflict are indicators for completing the design without failure. However, a process that allows designers to complete the design with ease is not necessarily a "good" process because it sometimes becomes difficult to reflect the customers' requirements. Such a problem occurs if an important attribute that is closely related to a customer's requirements has to be determined under several constraints in the derived process. In order to evaluate the ease with which the customer requirements and technological requirements can be reflected in the design, this research introduces another indicator, namely, "design difficulty of the important attribute." Fig. 6 shows an example of

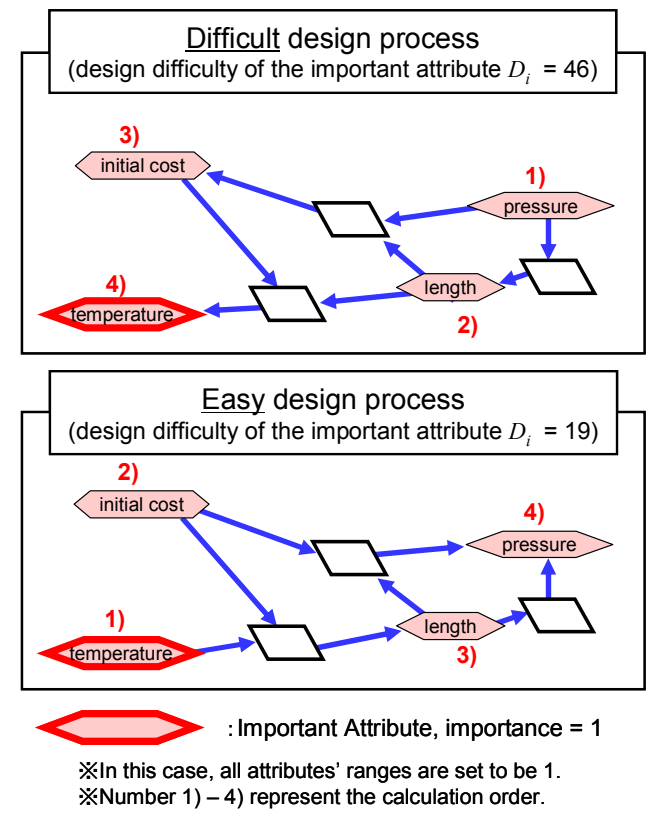

Fig. 6 Problem of reflecting important attributes 
design difficulty of the important attribute.

$$
D_{i}=\sum_{i}^{n^{a l l}} \frac{a^{i m p}{ }_{i} \times c^{c a l c}{ }_{i}}{r_{i}}
$$

where:

$D_{i}$ : design difficulty of the important attribute,

$a^{i m p}{ }_{i}$ importance of attribute $i$,

$a^{\text {calc }}$ : calculation order of attribute $i$,

$r_{i}$ : range of attribute $i$,

$n^{\text {all }}$ : number of attributes in the entire product system.

In this research, designers assigned values to each attribute importance; these values ranged from 1 to 10 . The higher the importance number of the attribute, the more important is the attribute. Because the objective of this research is the design of large-scale products, the number of related attributes is expected to be quite large. As a result, if the important attribute is calculated later, the design difficulty of the important attribute becomes quite large. If the design difficulty of the important attribute is small, it implies that the design process can easily reflect the customer requirements.

\section{Design Example (OTEC Plant System) and Discussion}

In order to examine the validity of this template design support system, we apply it to an actual design problem, that is, the design of an ocean thermal energy conversion (OTEC) plant. We have selected an OTEC plant system because many of the elements in an OTEC plant are interconnected and it is difficult for a designer to derive an optimal design process that is suited to the various environments such as location and surrounding water temperature.

Figure 7 shows the working principle of OTEC. Electricity is generated from the temperature difference between surface water (relatively warm) and deep water (relatively cool). The working fluid is transformed into a gas and is warmed in the evaporator. The produced gas rotates the turbine, which generates electricity.

\subsection{Steps to apply prototype system}

The proposed template model and design method are implemented as a prototype design system using CINCOM Smalltalk Visualworks $7.4^{(12)}$. The main functions of the design system are shown in Fig. 8. The prototype design system has the following functions.

1) Selection of a knowledge template from the template list

2) Definition of customer requirements in the product definition window

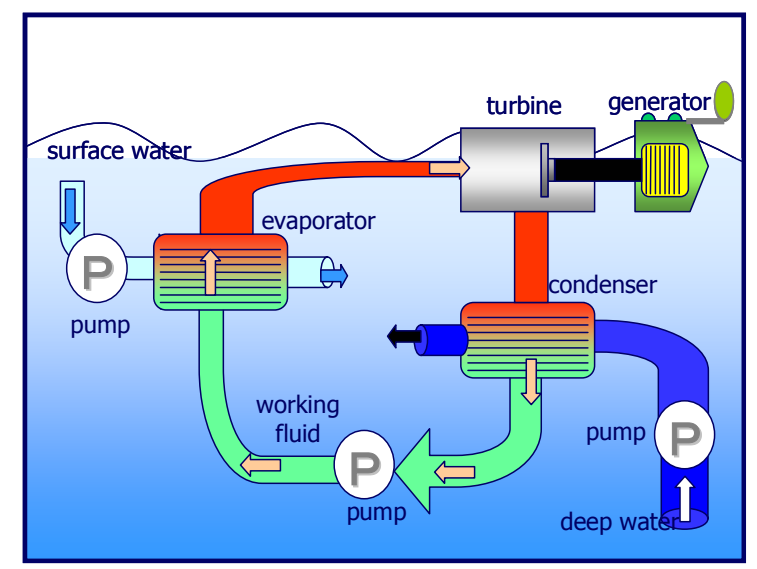

Fig. 7 Principle of OTEC ${ }^{13)}$ 




Fig. 8 Prototype management system of plant design

3) Generation, evaluation, and comparison of process candidates from the process list

4) Check of conflicts and loops in the current design situation

5) Execution of design tasks considering design problems by task list and problem manager.

A designer proceeds with the product design by following the five steps listed above. As an example, we assume certain conditions that must be solved in the OTEC design problem. The design requirements for the OTEC design include the electricity supply, which is the most important customer requirement (should be more than $2780 \mathrm{kWh}$ ) and the payback period (number of years required to collect the initial investment amount). The second requirement is important in itself, but it is less important than the first (electricity supply).

First, a designer describes the product information using the template model, which comprises the following elements: entity, interface, attribute, constraint, hierarchy link, and calculation flow.

Second, the designer describes the customer requirements by changing the importance of the relevant attributes or the boundary conditions, by setting the range for the relevant attributes. In the OTEC example, since the electricity supply is the most important attribute, the attribute "total electricity supply" is set to be the most important one.

Third, the designer selects the template to be used on the basis of the new product description. As mentioned previously, in this phase, the criterion used for selecting the template is consistency.

Fourth, the system automatically extracts the available constraints from the template and produces candidate processes.

These candidate processes are evaluated using Eqs. (1) (4) and plotted in a three-dimensional solution space (Fig. 9). The loop difficulty, conflict difficulty, and design difficulty of the important attribute are all normalized. In this prototype system, the 
recommended process is the one that is closest to the origin (circled in the figure). Finally, we proceed with the selected process. In this problem, the designer is supposed to use trial and error until the important attribute (i.e., electricity supply) has the largest value. Further, every attribute value must lie within the specified range in the final condition.

Figure 10 shows the results obtained for the OTEC design example using the proposed process and the existing process.

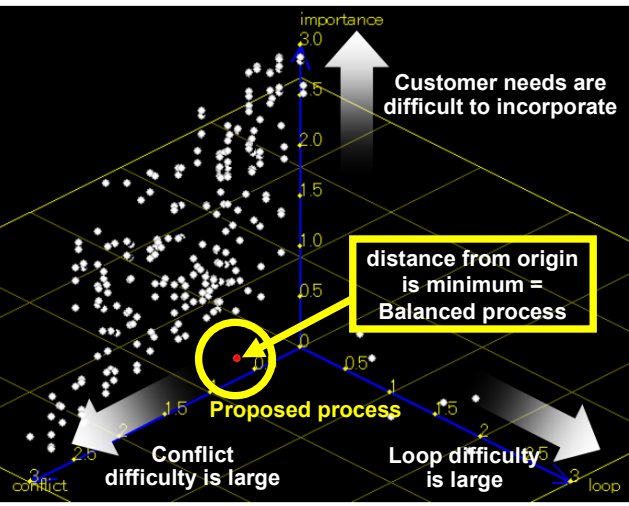

Fig. 9 Solution space of design process candidates As shown in Fig. 10, the most important attribute - "total electricity supply"-in the proposed process is better than that in the existing process, although the other attributes in the proposed process, such as "payback period" and "total initial cost," are lower than those in the existing process.

Figure 11 provides an overview of the existing OTEC process (each column is an attribute name and an entity name the attribute belongs to. A designer determines the values from the attributes in the upper column). In the existing process, the most important attribute - total electricity supply-has several constraints and it is thus difficult for a designer to reflect the customer requirements. In addition, attributes with a small range, such as "surface water quantity," are bound by several constraints, which leads to a high possibility of process failure.

On the other hand, it is easy to incorporate the requirements in the recommended process (Fig. 12) because a designer can decide the value of the important attributes without any constraints. Additionally, attributes with a small range have no constraints.

Since some, but not all, constraints are reversible, there is a possibility that a solution obtained using the existing process is better than that obtained from the recommended process. ("Constraints are reversible" implies that the input and output attributes can be exchanged.) On the basis of the design results, we can infer that the recommended process is more likely to yield a "good" process that can easily reflect the customer requirements and that has a small possibility of failure.

\begin{tabular}{|c|c|c|c|c|c|c|c|}
\hline & attribute & importance & valueMax & valueMin & output & unit & Range \\
\hline \multirow{10}{*}{$\begin{array}{l}\text { proposed } \\
\text { process }\end{array}$} & payback period@customer needs & 4 & 15 & 0 & 12.1 & year & 2 \\
\hline & total production of electricity@generating ability & 10 & 3430 & 2687 & 3008 & kWh & 0.24 \\
\hline & production of electricity@electric turbine & 7 & 4139 & 3039 & 4224 & kWh & 0.31 \\
\hline & flux@pipe for surface water & 1 & 5 & 4.2 & 5 & $\mathrm{~m} \hat{3} / \mathrm{s}$ & 0.17 \\
\hline & running cost@financial ability & 1 & 18.4 & 13.6 & 16.3 & M\$ & 0.3 \\
\hline & total initial cost@financial ability & 1 & 6000 & 4000 & 5972 & M\$ & 0.4 \\
\hline & tail water temperature@disposal part for surface water & 1 & 12 & 10 & 11.47 & ${ }^{\circ} \mathrm{C}$ & 0.18 \\
\hline & generating efficiency@electric turbine & 2 & 0.0035 & 0.0015 & 0.0026 & & 0.8 \\
\hline & & & & 4 & & & \\
\hline & attribute & importance & valueMax & valueMin & output & unit & Range \\
\hline \multirow{8}{*}{$\begin{array}{l}\text { existing } \\
\text { process }\end{array}$} & payback period@customer needs & 4 & 15 & 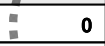 & 11.5 & yeâr & $2 \quad 2$ \\
\hline & total production of electricity@generating ability & 10 & 3430 & 2687 & 2689 & kWh & 0.24 \\
\hline & production of electricity@electric turbine & 7 & 4139 & $=3039$ & 3416 & kWh & 0.31 \\
\hline & flux@pipe for surface water & 1. & 5 & 4.2 & 5 & $\mathrm{~m}^{\wedge} 3 / \mathrm{s}$ & 0.17 \\
\hline & running cost@financial ability & $\because 1$ & 18.4 & 13.6 & 15.4 & $\mathrm{M} \$$ & 0.3 \\
\hline & total initial cost@financial ability & 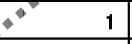 & 6000 & 4000 & 5112 & $M \$$ & 0.4 \\
\hline & tail water temperature@disposal part for surface water & 1 & 12 & 10 & 10 & ${ }^{\circ} \mathrm{C}$ & 0.18 \\
\hline & generating efficiency@electric turbine & 2 & 0.0035 & 0.0015 & 0.0027 & 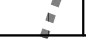 & 0.8 \\
\hline
\end{tabular}

Fig. 10 Comparison of design results obtained using the proposed process and the existing process 


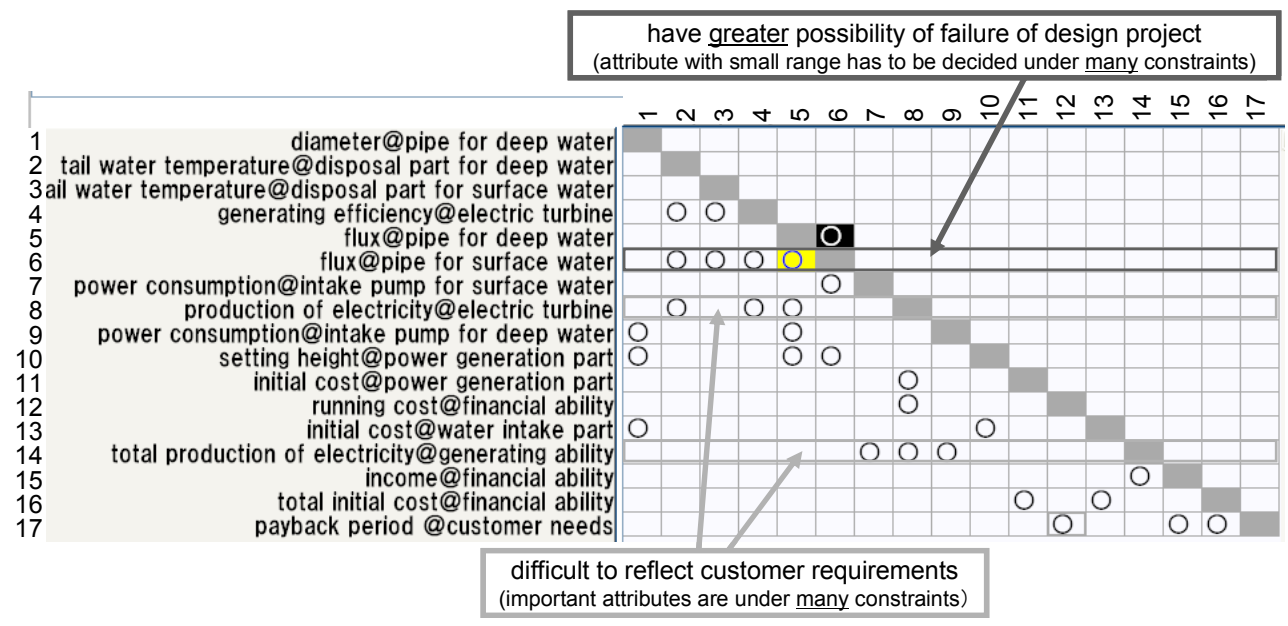

Fig. 11 Existing design process for an OTEC system

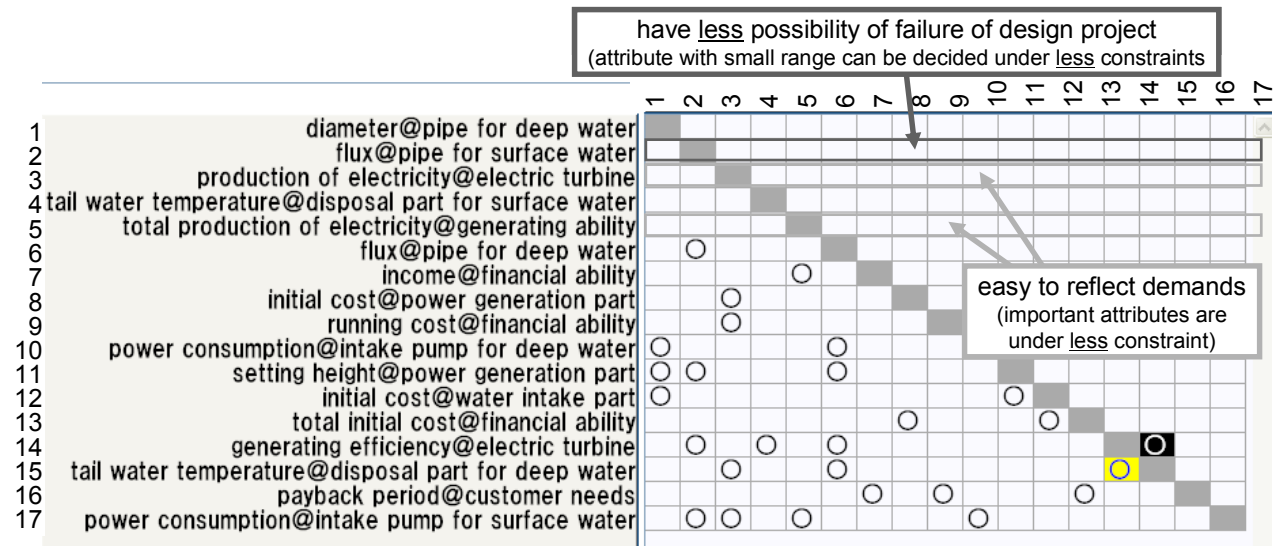

Fig. 12 Process recommended by the proposed method

\section{Concluding Remarks}

\subsection{Conclusion}

From the results of the implementation of the prototype system and the design of the OTEC plant system, we can conclude that the design knowledge for an actual OTEC design is well represented by the proposed knowledge template. Based on described knowledge template, the template design support system could also define a design process for an OTEC plant.

In order to confirm the adaptability of the recommended design process, the OTEC plant system was designed using two different design processes (the proposed process and the existing process). The most important attribute in the proposed design process has a higher achievement level than that in the existing process. The proposed process yields a good result because of the following two reasons. First, the existing design process has a greater possibility of failure than the proposed process since some attributes with a small range has to be decided under several constraints. Second, it is difficult to incorporate the customer requirements in the existing design process since important attributes have to be decided toward the end of the design process. From these design results and process characteristics, we can conclude that the proposed plant design management system provides a good design process in which customer requirements can be incorporated with ease. 


\subsection{Future works}

A knowledge template that utilizes not only the process knowledge but also the entity or interface knowledge is necessary. Such a template would support configuration design. In addition, the template model developed in this study could only be applied to improve upon an existing product; this is because if the calculated consistency between the new product description and the template data is too small, sufficient knowledge required to develop new processes cannot be collected. A template model that can be used to support completely new products would be necessary.

\section{References}

(1) Muchnick, H., Allen, J., McDowell, D., and Mistree, F., A Template-Based Approach For Multilevel Blast Resistant Panel Design, Proceedings of the ASME 2007 International Design Engineering Technical Conferences \& Computers and Information in Engineering Conference, DETC2007-35447

(2) Abbott, D. and Lough, K., Comparing Component Functional Template Modeling Experimental Results in an Undergraduate Level Engineering Design Course, Proceedings of the ASME 2008 International Design Engineering Technical Conferences \& Computers and Information In Engineering Conference, DETC2008-49349.

(3) Abbott, D. and Lough, K., Representing Historically Based Component-Function Relationships Through Design Templates, Proceedings of the ASME 2007 International Design Engineering Technical Conferences \& Computers and Information in Engineering Conference, DETC2007-35447.

(4) Otomi, K., Value Design: Trade off Between Value and Cost, Proceedings of the 14th JSME Design \& Systems Division, pp. 266-267.

(5) Koga, T. and Aoyama, K.: Design Process Guide Method for Minimizing Loops and Conflicts, Journal of Advanced Mechanical Design, Systems, and Manufacturing, Vol.3, No.3 (in press).

(6) Browning, T.R., Applying the Design Structure Matrix to System Decomposition and Integration Problems: A Review and New Directions, IEEE Transactions on Engineering Management, Vol.48, No.3 (2001), pp.292-306.

(7) Lévárdy, V. and Browning, T.R., An Adaptive Process Model to Support Product Development Project Management, IEEE Transactions on Engineering Management, forthcoming, 2009.

(8) Sosa M.E., Eppinger, S.D., and Rowles, C.M.: Identifying Modular and Integrative Systems and Their Impact on Design Team Interactions, Journal of Mechanical Design, Vol. 125, Issue 2 (2003), 240-253.

(9) Giffin, M., Weck, O., Bounova, G., Keller, R., Eckert, C., and Clarkson, P.J.: Change Propagation Analysis in Complex Technical Systems, Journal of Mechanical Design, Vol. 131, Issue 8 (2009), 081001 (14 pages).

(10) Wang, N.: ERMM: An Engineering Requirements Management Method, Journal of Computing and Information Science in Engineering, Vol. 6, Issue 2, (2006), 196-200.

(11) Yoshikawa, S., Systematization of Design Problems by Graph Theory, Proceedings of the International Design Conference - Design 2004, Dybrovnik, May 18 - 21, 2004.

(12) Visualworks (CINCOM Smalltalk), www.cincomsmalltalk.com

(13) Xenesys Inc.: http://www.xenesys.com/english/index.html 